\title{
Testing liquidity constraints in 10 Asian developing countries: an error-correction
} model approach

\begin{abstract}
An error-correction model is used to estimate the fraction of consumers who are liquidityconstrained in 10 Asian developing countries. Our estimates of the fraction of consumers who are liquidity-constrained range between 0.25 and 0.98 . We further investigate whether financial liberalization has resulted in the reduction of liquidity constraints in these countries. However, the results find support for this only in the cases of South Korea, Sri Lanka and Taiwan.
\end{abstract}

Keyword: error correction; finance; model test; neoliberalism; testing method 